Review Article

\title{
Superoxide Dismutase 1 Loss Disturbs Intracellular Redox Signaling, Resulting in Global Age-Related Pathological Changes
}

\author{
Kenji Watanabe, ${ }^{1,2}$ Shuichi Shibuya, ${ }^{1}$ Yusuke Ozawa, ${ }^{1}$ Hidetoshi Nojiri, ${ }^{3}$ Naotaka Izuo, \\ Koutaro Yokote, ${ }^{2}$ and Takahiko Shimizu ${ }^{1}$ \\ ${ }^{1}$ Department of Advanced Aging Medicine, Chiba University Graduate School of Medicine, Chiba 260-8670, Japan \\ ${ }^{2}$ Department of Clinical Cell Biology and Medicine, Chiba University Graduate School of Medicine, Chiba 260-8670, Japan \\ ${ }^{3}$ Department of Orthopaedics, Juntendo University Graduate School of Medicine, Bunkyo-ku, Tokyo 113-0033, Japan
}

Correspondence should be addressed to Takahiko Shimizu; shimizut@chiba-u.jp

Received 23 April 2014; Revised 29 July 2014; Accepted 6 August 2014; Published 8 September 2014

Academic Editor: Chi-Feng Hung

Copyright (c) 2014 Kenji Watanabe et al. This is an open access article distributed under the Creative Commons Attribution License, which permits unrestricted use, distribution, and reproduction in any medium, provided the original work is properly cited.

\begin{abstract}
Aging is characterized by increased oxidative stress, chronic inflammation, and organ dysfunction, which occur in a progressive and irreversible manner. Superoxide dismutase (SOD) serves as a major antioxidant and neutralizes superoxide radicals throughout the body. In vivo studies have demonstrated that copper/zinc superoxide dismutase-deficient (Sod1 ${ }^{-/-}$) mice show various aginglike pathologies, accompanied by augmentation of oxidative damage in organs. We found that antioxidant treatment significantly attenuated the age-related tissue changes and oxidative damage-associated p53 upregulation in Sod1 ${ }^{-/-}$mice. This review will focus on various age-related pathologies caused by the loss of Sod1 and will discuss the molecular mechanisms underlying the pathogenesis in $\mathrm{Sodl}^{-1-}$ mice.
\end{abstract}

\section{Introduction}

Aging is associated with several functional and structural deficits in organs, which are linked to biochemical changes, including oxidative modifications, protein aggregation, and altered gene expression [1]. Reactive oxygen species (ROS) are mainly generated from the electron transport chain in mitochondria and nonspecifically oxidize cellular molecules such as proteins, nucleic acids, and lipids, thus resulting in the accumulation of oxidative damage in organisms [2].

The redox balance is physiologically regulated through the production and degradation of ROS in antioxidant systems to protect cells from oxidative damage. Superoxide dismutase (SOD) enzymes play a major role in the antioxidant system by catalyzing the conversion of superoxide radicals $\left(\mathrm{O}_{2}{ }^{-}\right)$to hydrogen peroxide $\left(\mathrm{H}_{2} \mathrm{O}_{2}\right)$ and $\mathrm{O}_{2}$ [3]. In mammals, there are three SOD isoforms: CuZn-SOD (Sod1), which exists in the cytoplasm; Mn-SOD (Sod2), which is distributed in the mitochondrial matrix; and extracellular SOD (Sod3), which is localized in extracellular fluids, such as lymph, synovial fluid, and plasma.
Mice lacking Sod2 showed dilated cardiomyopathy, steatosis, and metabolic acidosis, which resulted in neonatal lethality [4]. Therefore, heterozygous $\left(\mathrm{Sod}^{+/-}\right)$knockout or tissue-specific knockout mice are used to analyze the physiological role of Sod2 in various tissues and organs $[5,6]$. Carlsson et al. generated Sod3-null mutant mice [7]. Although Sod $3^{-/-}$mice exhibited a shorter survival time than wild-type controls under hyperoxic conditions, the mice grew with no apparent abnormalities until late in life. In contrast, Reaume et al. first described the characterization of global Sod1-deficient $\left(\right.$ Sod1 $\left.^{-/-}\right)$mice. These mice exhibited marked vulnerability to motor neuron loss after axonal injury [8]. Subsequently, Sod1 ${ }^{-/-}$mice showed a significantly shortened mean lifespan by approximately $30 \%$ and a high incidence of liver tumors by 20 months of age compared with those of $\mathrm{Sodl}^{+/+}$mice [9]. In vitro studies also revealed that Sod1 ${ }^{-/-}$ fibroblasts showed a significantly decreased growth rate and higher sensitivity to $\mathrm{O}_{2}$ stress than $\mathrm{Sodl}^{+/+}$cells [10]. In the following paragraphs, we will introduce the various organ and tissue changes associated with the cellular phenotypes in Sodl ${ }^{-/-}$mice. 
TABLE 1: The age-related pathologies in $\mathrm{Sodl}^{-/-}$mice.

\begin{tabular}{llc}
\hline Brain & Acceleration of Alzheimer's disease & {$[11,12]$} \\
Eye & Macular degeneration & {$[13,14]$} \\
& Cataract & {$[15]$} \\
& Dry eye & {$[16,17]$} \\
Ear & Cochlear hair cell loss & {$[18]$} \\
& Hearing loss & {$[19]$} \\
Blood & Hemolytic anemia & {$[20]$} \\
Bone & Osteopenia & {$[21,22]$} \\
Skin & Skin atrophy & {$[23,24]$} \\
Muscle & Skeletal muscle atrophy & {$[25]$} \\
Pancreas & Glucose intolerance & {$[26,27]$} \\
Liver & Hepatocellular carcinoma & {$[9]$} \\
& Fatty deposits & {$[28]$} \\
Ovary & Infertility & {$[29,30]$} \\
& Luteal degeneration & {$[31]$} \\
\hline
\end{tabular}

\section{Sod1 ${ }^{-/-}$Mice Exhibit Age-Related Pathological Changes in Various Organs and Tissues}

We and other groups have demonstrated that Sod1 ${ }^{-/-}$mice show various aging-like tissue changes, such as acceleration of Alzheimer's disease (AD) [11, 12], macular degeneration $[13,14]$, cataracts $[15]$, dry eye $[16,17]$, cochlear hair cell loss [18], hearing loss [19], hemolytic anemia [20], osteopenia $[21,22]$, skin atrophy $[23,24]$, skeletal muscle atrophy [25], glucose intolerance [26, 27], hepatic carcinoma [9], fatty liver [28], infertility [29, 30], and luteal degeneration [31] (Table 1). Furthermore, the biochemical analyses revealed that Sod1 loss in organs led to the accumulation of oxidative molecules such as carbonylated proteins, lipid peroxidants, oxidized nucleic acids, and advanced glycation end products (AGEs), which resulted in broadly impaired cellular signaling, gene expression, energy metabolism, cytoskeletal morphology, and cell death in the tissues.

\section{Effects on the Individual Organs and Tissues}

3.1. Effects on the Brain. Brain function declines in patients with neurodegenerative diseases, as well as during normal aging [32]. Ansari and Scheff reported a strong correlation between oxidative damage levels (total SOD, glutathione, catalase, thiobarbituric acid reactive substances, protein carbonyl, 3-nitrotyrosine, 4-hydroxynonenal, and acrolein) and the variable dementia status of subjects [33]. In addition, we have previously reported a specific reduction of SOD1 protein level, but not SOD2 and SOD3, in neocortex of AD brains [11]. We also reported that a mouse model for AD lacking Sod1 showed exacerbation of memory loss and behavioral abnormalities associated with accelerated plaque formation and amyloid accumulation [11, 12]. Furthermore, a biochemical analysis also revealed high levels of intracellular $\mathrm{N} \varepsilon$-(carboxymethyl) lysine (CML) and 8-hydroxy-2' deoxyguanosine $(8-\mathrm{OHdG})$ in the mouse brain. In addition,
Sod1 deficiency induced neuronal inflammation, as demonstrated by astrocyte and microglial activation in a mouse model for AD. These findings strongly suggested that SOD1 expression plays a pivotal role in maintaining cellular redox balance and brain function during aging.

3.2. Effects on the Eyes. Several eye diseases, such as agerelated macular degeneration, cataracts, dry eye, phacoemulsification, and presbyopia, are closely related to the aging process $[32,34]$. Sod1 deficiency induced the development of drusen-like deposits in the retina, choroidal neovascularization, and retinal pigment epithelium dysfunction, thus resulting in age-related retinal degenerative disorders, including age-related macular degeneration $[13,14]$. An immunohistochemical analysis also revealed that CML-positive deposits were abundantly detected in the retinas of aged Sod1 ${ }^{-/-}$mice [13]. Moreover, the Sod1 ${ }^{-/-}$mouse lens showed twice the level of $\mathrm{O}_{2}{ }^{--}$generation compared with that of control mice and had accelerated cataractogenesis following ultraviolet irradiation [15]. Furthermore, Dogru and colleagues reported that Sod1 $1^{-1-}$ mice also exhibited typical dry eye associated with lacrimal gland and meibomian gland changes, and this occurred in an age-dependent manner [16, 17]. The Sod1 $1^{-/-}$lacrimal and meibomian glands showed increased 4-hydroxy-2-nonenal and 8-OHdG staining, apoptotic cells, and inflammatory infiltrates at 50 weeks of age compared to $\mathrm{Sodl}^{+/+}$mice. In addition, electron microscopy observations detected ultrastructural alterations in the mitochondria, including swelling, disorientation, shortening, disorganized cristae, marked fragmentation, shrinkage of the nuclei, and cytoplasmic vacuole formation, as well as the loss of nuclear membranes in Sod1 ${ }^{-/-}$mice.

3.3. Changes in the Ears. The cochlear structure in the ear is progressively degenerated during aging, leading to hearing loss $[35,36]$. McFadden et al. reported that Sod1 deficiency morphologically induced a reduction of the inner and outer hair cells during aging [18]. In addition, Sod1 ablation impacted the noise-induced permanent threshold shifts, leading to hearing loss $[18,19]$. On the other hand, systemic overexpression of human Sod1 protected against age-related and noise-induced hearing loss in C57BL/6 mice [37].

3.4. Changes in the Blood. During aging, the levels of oxidative stress markers, including 8-isoprostane and 2thiobarbituric acid reactive substances (TBARS), are gradually increased in the plasma and erythrocytes of Sod $1^{-/-}$mice [20]. Furthermore, Iuchi et al. reported that an intracellular ROS indicator, $\mathrm{CM}-\mathrm{H}_{2} \mathrm{DCFDA}$ (DCF), in erythrocytes was spontaneously elevated in Sod1 ${ }^{-/-}$mice. Sod1 $1^{-1-}$ mice also showed hemolytic anemia associated with splenomegaly. In fact, the erythrocyte lifespan from Sod1 $1^{-1-}$ mice was decreased by $60 \%$ compared to that of Sod $1^{+/+}$erythrocytes [20]. We independently measured the serum levels of various markers of inflammation in $\mathrm{Sodl}^{-/-}$ 
TABLE 2: The serum biomarker levels in Sod1 ${ }^{-1-}$ mice.

\begin{tabular}{|c|c|c|c|c|}
\hline Markers & Concentrations & Sod1 $1^{+/+}$ & Sod1 $1^{-1-}$ & $P$ value \\
\hline IL-10 & $\mathrm{ng} / \mathrm{mL}$ & $425 \pm 54$ & $451 \pm 60$ & 0.495 \\
\hline IL-11 & $\mathrm{pg} / \mathrm{mL}$ & $39 \pm 14.4$ & $33 \pm 13.2$ & 0.627 \\
\hline IL-12p70 & $\mathrm{ng} / \mathrm{mL}$ & ND & ND & - \\
\hline IL-17 & $\mathrm{pg} / \mathrm{mL}$ & ND & ND & - \\
\hline IL-18 & $\mathrm{ng} / \mathrm{mL}$ & $10 \pm 1.1$ & $12 \pm 1.21$ & 0.105 \\
\hline IL-1alpha & $\mathrm{pg} / \mathrm{mL}$ & $83 \pm 63$ & $134 \pm 89.4$ & 0.467 \\
\hline IL-1beta & $\mathrm{ng} / \mathrm{mL}$ & $12 \pm 1.1$ & $13 \pm 1.6$ & 0.268 \\
\hline IL-2 & $\mathrm{pg} / \mathrm{mL}$ & ND & ND & - \\
\hline IL-3 & $\mathrm{pg} / \mathrm{mL}$ & ND & ND & - \\
\hline IL-4 & $\mathrm{pg} / \mathrm{mL}$ & $20.2 \pm 0.0$ & $20.2 \pm 0.0$ & 1 \\
\hline IL-5 & $\mathrm{ng} / \mathrm{mL}$ & $0.23 \pm 0.066$ & $0.19 \pm 0.055$ & 0.406 \\
\hline IL-6 & $\mathrm{pg} / \mathrm{mL}$ & $4.4 \pm 1.7$ & $18 \pm 14.3$ & 0.102 \\
\hline IL-7 & $\mathrm{ng} / \mathrm{mL}$ & $0.02 \pm 0.016$ & $0.05 \pm 0.025$ & 0.296 \\
\hline IP-10 & $\mathrm{pg} / \mathrm{mL}$ & $99 \pm 17.1$ & $150 \pm 60.3$ & 0.109 \\
\hline M-CSF & $\mathrm{pg} / \mathrm{mL}$ & $6.9 \pm 0.50$ & $6.0 \pm 0.25$ & $0.010^{*}$ \\
\hline MCP-1 & $\mathrm{pg} / \mathrm{mL}$ & $100 \pm 34.2$ & $124 \pm 60.0$ & 0.457 \\
\hline MCP-3 & $\mathrm{pg} / \mathrm{mL}$ & $235 \pm 50.9$ & $235 \pm 69.3$ & 0.996 \\
\hline MCP-5 & $\mathrm{pg} / \mathrm{mL}$ & $18 \pm 1.7$ & $24 \pm 7.0$ & 0.094 \\
\hline MIP-1alpha & $\mathrm{ng} / \mathrm{mL}$ & $1.6 \pm 0.21$ & $1.7 \pm 0.09$ & 0.307 \\
\hline MIP-1beta & $\mathrm{pg} / \mathrm{mL}$ & $55 \pm 16.5$ & $80 \pm 17.5$ & $0.005^{*}$ \\
\hline MIP-Igamma & $\mathrm{pg} / \mathrm{mL}$ & $26 \pm 3.6$ & $34 \pm 3.7$ & $0.013^{*}$ \\
\hline MIP-2 & $\mathrm{pg} / \mathrm{mL}$ & $18 \pm 2.1$ & $20 \pm 5.2$ & 0.371 \\
\hline MIP-3beta & $\mathrm{ng} / \mathrm{mL}$ & $1.8 \pm 0.37$ & $1.7 \pm 0.14$ & 0.349 \\
\hline MDC & $\mathrm{pg} / \mathrm{mL}$ & $547 \pm 234$ & $626 \pm 42$ & 0.481 \\
\hline RANTES & $\mathrm{pg} / \mathrm{mL}$ & $0.26 \pm 0.130$ & $0.45 \pm 0.049$ & $0.014^{*}$ \\
\hline TNF-alpha & $\mathrm{ng} / \mathrm{mL}$ & $0.066 \pm 0.004$ & $0.077 \pm 0.010$ & $0.041^{*}$ \\
\hline TPO & $\mathrm{ng} / \mathrm{mL}$ & $75 \pm 9.4$ & $86.5 \pm 5.6$ & $0.049^{*}$ \\
\hline
\end{tabular}

ND indicates "not detected". *indicates a significant difference.

mice. A multiplex analysis revealed an altered pattern of inflammation markers, such as macrophage colony stimulating factor (M-CSF), macrophage inflammatory protein-1 beta (MIP-1 beta), macrophage inflammatory protein-1 gamma (MIP-1 gamma), regulated on activation, normal $\mathrm{T}$ cell expressed and secreted (RANTES), tumor necrosis factor-alpha (TNF-alpha), and thrombopoietin (TPO) in the Sod1 $1^{-/-}$mouse sera (Table 2).

3.5. Effects on Bone. Aging stress generally causes bone loss and fragility [38]. We previously clarified that the loss of Sod1 caused bone loss without leading to developmental skeletal abnormalities in both male and female mice [21]. The threedimensional computed tomography analyses revealed that there was marked bone loss in both cortical and cancellous bones of Sod1 $1^{-/-}$mice, which was associated with decreased bone formation and resorption, indicating the presence of low-turnover osteopenia (Figure 1). Sod1 deficiency also enhanced the intracellular ROS production and the formation of pentosidine, one of the AGEs, in osteoblasts and bone [21]. Furthermore, Wang et al. also reported that young Sod $1^{-/-}$mice showed bone fragility in the femora at the growth stage [39].
Recently, we found that mechanical unloading-induced bone loss associated with intracellular ROS generation in bone-forming cells and bone marrow cells [22]. Interestingly, we also detected specific Sod1 upregulation at both the RNA and protein levels in bone during mechanical unloading [22]. Notably, Sod1 deficiency significantly exacerbated the bone loss during mechanical unloading. In addition, Sod1 $1^{-/-}$ mice clearly displayed four-layered structural abnormalities and fragmented tidemarks in the enthesis, indicating tendon enthesis degeneration [40]. These findings suggested that Sod1 plays a protective role in regulating bone and tendon enthesis homeostasis, as well as the redox balance during unloading and aging in mice.

3.6. Changes in the Skin. Aged skin is characterized by wrinkles, sagging, dryness, and collagen degradation $[41,42]$. We have previously reported that Sod1 deletion caused typical age-related skin thinning [23]. In hematoxylin and eosin stained sections, the epidermis and dermis of the Sod1 $1^{-1-}$ back skin showed remarkable thinning (Figure 2(a)). In addition, the skin weight and hydroxyproline content, which is a unique amino acid present in collagen and elastin, in the Sod1 $1^{-/-}$mice were compared with those of Sod1 ${ }^{+/+}$mice [24]. 

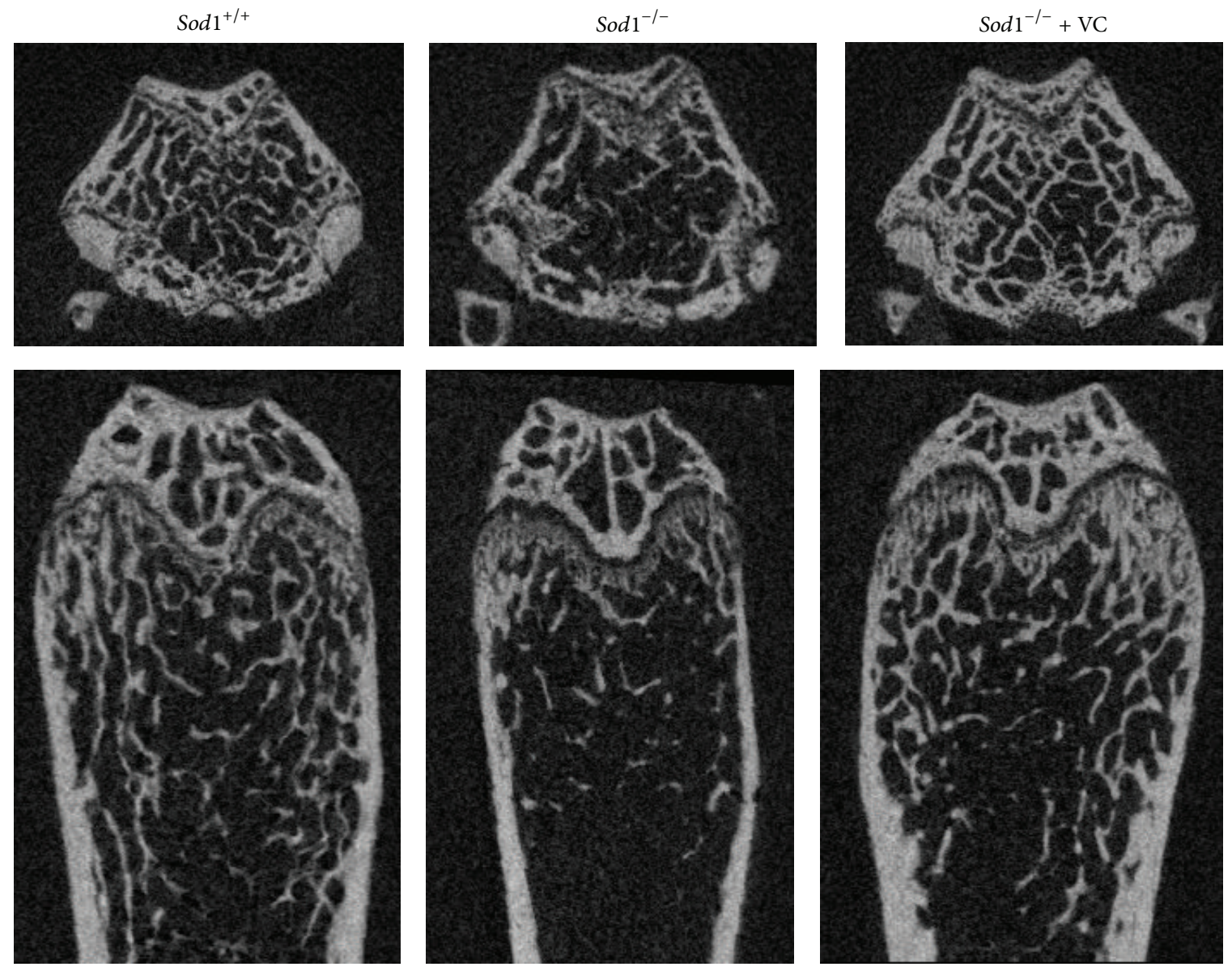

FIgure 1: The bone loss in Sod1 ${ }^{-/-}$mice. The treatment with $1 \%$ vitamin C in drinking water started from 4 weeks of age and continued for 12 weeks. Axial (upper panels) and coronal (lower panels) sections of $\mu \mathrm{CT}$ images of the distal ends of the femora of Sod1 $1^{+/+}$and Sod1 ${ }^{-/-}$females at 16 weeks of age.

An in vitro analysis using primary dermal fibroblasts from Sod1 $1^{-/-}$neonates revealed severe cellular phenotypes, such as apoptosis and growth arrest, under normal conditions (Figure 2(b)). Furthermore, Sod1 ${ }^{-/-}$fibroblasts showed excessive intracellular DCF-positive fluorescence (Figure 2(c)). Interestingly, Sod1 ${ }^{-/-}$fibroblasts also had a significant enhancement of mitochondrial $\mathrm{O}_{2}{ }^{--}$and impairment of the mitochondrial membrane potential [43].

3.7. Effects on Muscle. Aging contributes to the structural and functional changes in skeletal muscle in a wide range of mammals [44]. Sod1 ${ }^{-/-}$mice showed significant decreases in the whole hindlimb muscle mass compared with agematched Sod $1^{+/+}$mice, and this occurred in an age-dependent manner [25]. A biochemical analysis also revealed a significant increase in oxidative damage, such as the formation of F2-isoprostanes, protein carbonyls, and 8-OHdG, in Sod1 ${ }^{-1-}$ skeletal muscle [25]. Sod1 loss also induced aberrant mitochondria with abnormal shapes and led to lower ATP production in muscle. Mitochondria isolated from Sod1 $1^{-1-}$ muscle revealed significant increases in $\mathrm{O}_{2}{ }^{--}$ and $\mathrm{H}_{2} \mathrm{O}_{2}$ production and no compensatory upregulation of other antioxidant enzymes [45]. Recently, Zhang et al. reported that skeletal muscle-specific Sod1 ${ }^{-1-}$ mice failed to show muscle loss and ROS production [46]. Interestingly, a neuron-specific Sod1 transgene in Sod1 ${ }^{-/-}$mice prevented muscle loss [47]. The muscle from Sod1 ${ }^{-/-}$mice with a brain-specific Sod1 transgene did not show any differences in the muscle morphology, function, lipid peroxidation, or protein nitration compared with those of Sod1 ${ }^{+/+}$muscle, suggesting that Sod 1 insufficiency in neuronal cells could lead to a dysregulation of the muscle mass and function in a nonautonomous manner.

3.8. Effects on the Pancreas. Aging stress also impairs insulin secretion and sensitivity in the pancreas [48]. Wang et al. reported that Sod $^{-/-}$islets exhibited a decreased $\beta$-cell mass, impaired glucose-stimulated insulin secretion, and a decreased ATP content, accompanied by elevated intracellular ROS levels [26]. In addition, Sod1 ablation also downregulated the duodenal homeobox-1 $(P d x 1)$ expression and forkhead box protein A2 (Foxa2) pathway in an $\mathrm{O}_{2}{ }^{--}$-dependent fashion by affecting these targets at the epigenetic, mRNA, and protein levels in the islets [26]. Furthermore, Muscogiuri 

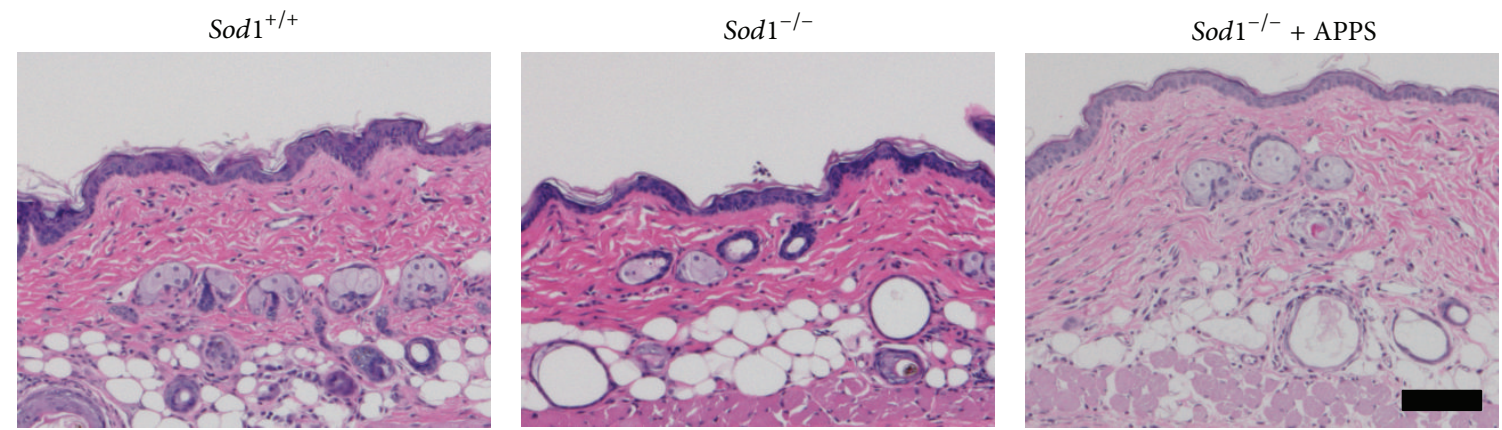

(a)
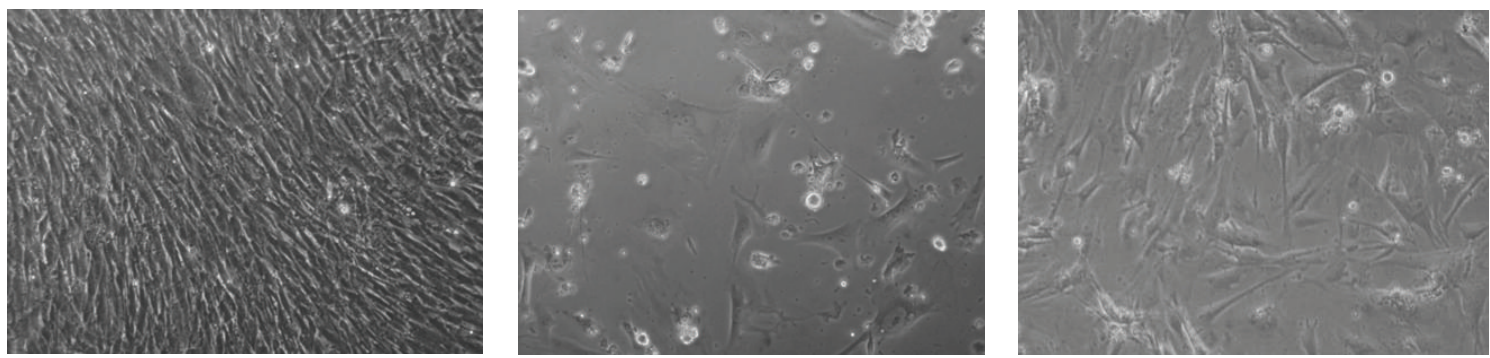

(b)
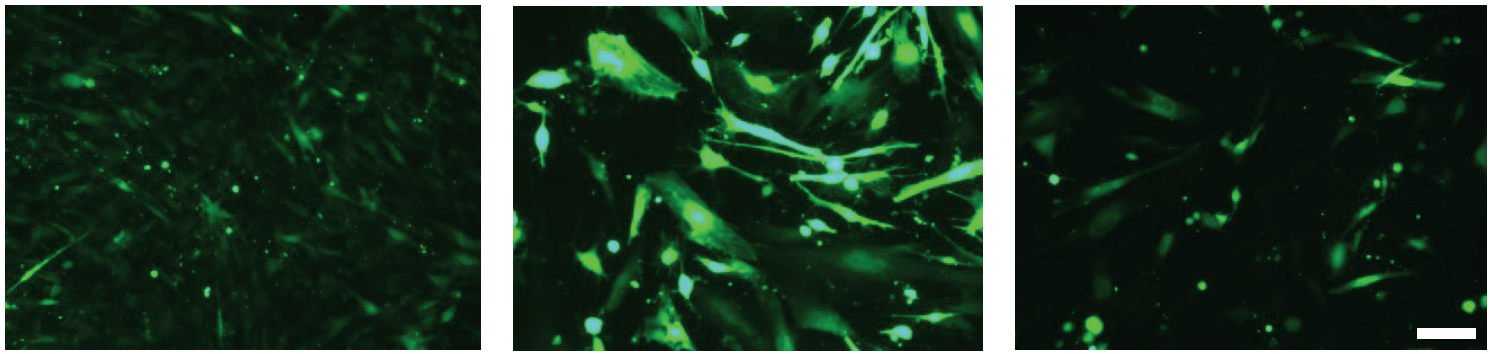

(c)

Figure 2: The skin and cellular phenotypes of Sod1 ${ }^{-/-}$mice. (a) The hematoxylin and eosin staining of the back skin of Sod1 ${ }^{-/-}$and Sod1 $1^{+/+}$ mice. Sod1 ${ }^{-1-}$ mice (5 months of age) were transdermally treated with $1 \% \mathrm{~L}$-ascorbyl 2-phosphate 6-palmitate trisodium salt (APPS) for 4 weeks. (b) Dermal fibroblasts were dissected from Sod1 ${ }^{-1-}$ neonates at 5 days of age. The cells were cultured with or without $10 \mu \mathrm{M}$ APPS for $48 \mathrm{~h}$ under $20 \% \mathrm{O}_{2}$. (c) The intracellular ROS levels in Sod1 ${ }^{-1-}$ fibroblasts treated with $10 \mu \mathrm{M}$ APPS were measured by examining the presence of CM- $\mathrm{H}_{2}$ DCFDA. The scale bars represent $100 \mu \mathrm{m}$.

et al. also showed that Sod1 loss significantly impaired the glucose tolerance and led to a reduced $\beta$-cell mass, as well as insulin secretion in a hyperglycemic clamp test [27]. Interestingly, Sod1 ablation failed to alter the peripheral and hepatic insulin sensitivity. These results proved that the absence of Sod 1 impaired $\beta$-cell function and glucose tolerance, but not insulin sensitivity, thus resulting in diabetes-like phenotypes.

3.9. Changes in the Liver. Aging of the liver is associated with an increased incidence of tumorigenesis [49]. The liver weight to body weight ratio in Sod1 $1^{-/-}$mice was significantly higher than that of Sod1 $1^{+/}$mice [9]. Sod1 $1^{-/-}$mice also showed increased oxidative damage such as malondialdehyde (MDA), F2-isoprostane, and 8-OHdG accumulation in their livers [9]. In addition, the Sod1 ${ }^{-/-}$livers showed an approximately $30 \%$ increase in hepatocarcinogenesis at 20 months of age compared to wild-type mice [9]. We also observed that Sod1 $1^{-/-}$mice showed significantly accelerated hepatic lipid accumulation and peroxidation and impaired lowdensity lipoprotein secretion due to apoB degradation that occurred via a posttranslational mechanism [28]. Furthermore, Wang et al. reported that Sod1 loss enhanced glycolysis and lipogenic signaling but decreased gluconeogenesis in the liver [50]. Recently, Kondo et al. described that the loss of senescence marker protein-30 (SMP30), which is a key enzyme required for L-ascorbic acid biosynthesis [51], accelerated the hepatic steatosis in Sod1 $1^{-/-}$mice [52]. Both Sod1 and Smp30 deficiency led to a remarkable elevation of the triglyceride and cellular $\mathrm{O}_{2}{ }^{--}$levels in the liver compared 
to those of Sod1 $1^{-/-}$or $S m p 30^{-/-}$mice. These findings indicated that elevated oxidative stress and/or L-ascorbic acid depletion altered the glucose and lipid metabolism in the liver, suggesting that normal SOD1 expression is essential to maintain the hepatic glucose and lipid homeostasis.

In pharmacological studies, acetaminophen (APAP) injection induces glutathione depletion, the formation of reactive nitrogen species, and plasma ALT elevation, resulting in lethal hepatotoxicity in the case of an overdose [53]. Interestingly, Sodl deficiency attenuated the APAP-induced hepatotoxicity and lethality owing to its reduction of hepatic APAP-cysteine adducts, protein nitration, and CYP2E1 activity, which acts as an APAP-metabolizing enzyme, in the liver $[53,54]$. These data indicated that the increases in intracellular $\mathrm{O}_{2}{ }^{--}$caused by Sod1 deletion inhibited CYP2E1 activity, thus resulting in protection against APAP-induced hepatotoxicity.

3.10. Effects on the Ovaries. Ovarian aging is characterized by a decline in the follicle numbers and sex steroid hormone secretion, which are associated with a gradual decline in fertility [55]. Although Sod1 ${ }^{-1-}$ female mice had normal estrous cycles and numbers of ovulated ova, their reproductive performance was inferior to that of female Sodl ${ }^{+/+}$and Sodl $1^{+/-}$ mice $[29,30]$. A hormonal analysis revealed that Sod1 ${ }^{-1-}$ females showed normal plasma levels of follicle-stimulating hormone (FSH), luteinizing hormone (LH), and estradiol at proestrus [31]. On the other hand, the plasma progesterone level was specifically repressed in Sod1 ${ }^{-1-}$ females compared to that in $\mathrm{Sodl}^{+/+}$females during pregnancy. Although Sodl loss in the ovaries and oocytes upregulated the intracellular ROS production, Sod1 ${ }^{-/-}$oocytes could be normally fertilized and developed to the two-cell stage in vitro $[31,56]$. However, Sodl ${ }^{-/-}$embryos failed to divide to the four-cell stage under conventional culture conditions $\left(20 \% \mathrm{O}_{2}\right)$ [56]. When SodI ${ }^{-/-}$embryos were cultured under hypoxic conditions (1\% $\mathrm{O}_{2}$ ), they developed to the morula stage but could not develop into blastocysts [56], indicating that $\mathrm{O}_{2}$ stress inhibited the development of Sod1 ${ }^{-/-}$embryos at the two-cell stage.

\section{Intervention Strategies Using Antioxidants}

Vitamin C (VC) is a soluble vitamin and the best characterized antioxidant [57]. In order to evaluate the protective effects of this antioxidant in Sodl ${ }^{-/-}$mice, we treated them with VC to try to rescue the organ phenotypes. Oral administration of VC suppressed the bone loss of $\mathrm{Sod1}^{-1-}$ mice, indicating that $\mathrm{O}_{2}{ }^{--}$-induced bone loss could be improved by antioxidant treatment (Figure 1) [21]. In addition, VC treatment also normalized the bone strength and composition of collagen cross-links, without aberrant bone modeling [21]. We further applied a VC derivative, Lascorbyl 2-phosphate 6-palmitate trisodium salt (APPS), on the Sod1 $1^{-1-}$ mouse skin. APPS is conjugated to a phosphate group and a long hydrophobic chain to promote stability and membrane permeability. The transdermal administration of the APPS reversed the skin atrophy and lipid peroxidation in Sod1 ${ }^{-/-}$mice (Figure 2(a)). In vitro experiments revealed that APPS treatment completely improved the cell viability and suppressed the intracellular ROS production in Sod1 ${ }^{-/}$ fibroblasts (Figures 2(b) and 2(c)). Furthermore, Iuchi et al. reported that oral N-acetyl cysteine (NAC) treatment attenuated the hemolytic anemia and inflammatory response, with ROS suppression, in the erythrocytes for Sod1 ${ }^{-1-}$ mice [20]. Additionally, we found that NAC treatment also improved the cell viability and decreased the intracellular ROS level in Sod1 ${ }^{-/-}$fibroblasts [43].

The oxidative stress induced by Sod 1 deficiency is closely related to the progression of $\mathrm{AD}$. Therefore, we hypothesized that antioxidant treatment would be able to alleviate the progression of $\mathrm{AD}$. In this context, we treated mice with AD-like pathologies with VC. Confirming our hypothesis, chronic VC treatment restored the behavioral abnormalities, apparently by attenuating the oxidative stress in $\mathrm{AD}$ model mice [58]. VC also significantly suppressed the soluble $A \beta$ accumulation in the brain, but not the plaque formation in the AD model mice [58]. Recently, we found that VC administration significantly prevented unloading-induced bone loss in wild-type mice [22]. These data strongly indicated that antioxidant intervention has remarkable protective effects against ROS-mediated tissue damage in mice.

\section{Molecular Mechanisms Underlying the Organ and Tissue Pathologies in Sod1 ${ }^{-/-}$Mice}

To analyze the molecular mechanisms underlying the tissue damage induced by Sod 1 deficiency, we have investigated the phenotypes using double-knockout Sod1 and liver-specific Sod2 mice. As described above, the Sod1 ${ }^{-/-}$mice showed acceleration of hepatic lipid accumulation, accompanied by increased oxidative damage. In contrast, liver-specific Sod2 knockout mice did not show any obvious morphological abnormalities or spontaneous oxidative damage in the liver [59]. The double-knockout mice had an indistinguishable hepatic phenotype, including lipid peroxidation, lipid accumulation, and TG secretion, from that of Sod1 ${ }^{-/-}$mice, indicating that the loss of Sod2 failed to exacerbate the hepatic changes in Sodl ${ }^{-1-}$ mice [28], demonstrating that the different enzymes do not have overlapping functions. Sentman et al. reported that combined Sod 1 and Sod 3 deficiency showed no additive effect on the lifespan and body weight in mice [60]. Likewise, Fujita et al. reported that Sod 1 and Sod3 double mutant mice showed the same phenotypes, such as $\mathrm{O}_{2}{ }^{-}$ and NO production and the TBARS level, in the kidneys compared with those of wild-type mice [61]. Moreover, glutathione peroxidase-1 (GPX1) loss also had no impact on the Sod1 ${ }^{-/-}$phenotypes in the liver and pancreas $[26,62]$. However, Sod1 loss significantly decreased the GPX1 activity, but not the Gpxl level in the liver. The Lei group reported that Sod1 loss increased the conversion of selenocysteine to dehydroalanine residues in the active site of hepatic GPX1, thus leading to proportional decreases in the activity of the enzyme as a whole [63]. Additionally, many reports have 
demonstrated that Sodl ${ }^{-/-}$mice showed no compensatory upregulation of antioxidant enzymes including $\operatorname{Sod} 2$ and Sod3 [43, 46, 60, 61]. These reports suggested that Sod2, Sod3, and/or Gpx1 deficiency failed to further modify the organ pathologies in Sod1 ${ }^{-/-}$mice.

Accumulating evidence suggests that both ataxiatelangiectasia mutated (ATM) and p53 play a central role in the DNA damage response induced by oxidative damage in organs and tissues [64]. In this context, Erker et al. investigated the organ phenotypes in mice lacking both Sod1 and Atm to elucidate DNA damage response in the organs. The loss of Atm and Sod 1 did not show any interaction with regard to the overall cellular metabolism and survival in mice [65], indicating that Sod1 regulates organ metabolism and lifespan in an Atm-independent manner.

Interestingly, we found that $\mathrm{Sodl}^{-1-}$ skin displayed obvious p53 activation [43]. Additionally, treatment with a VC derivative remarkably suppressed the $p 53$ expression and oxidative damage in the skin of Sodl $1^{-/-}$mice, suggesting that the antioxidant activity of $\mathrm{VC}$ normalized the skin pathologies, at least in part, by suppressing $\mathrm{O}_{2}{ }^{-{ }^{-}}$-mediated p53 activation in vivo [43]. Furthermore, the Sod1 loss induced the phosphorylation of $\mathrm{H} 2 \mathrm{AX}$ at Ser139 $(\gamma \mathrm{H} 2 \mathrm{AX})$, a DNA damage marker, and upregulated p21, a target gene of p53, in fibroblasts [43]. Of note, the Sod1 ${ }^{-/-}$fibroblasts exhibited a loss of mitochondrial membrane potential and enhanced mitochondria ROS generation. Likewise, Muller et al. reported that $S_{0} \mathrm{dl}^{-/-}$skeletal muscle showed significant alterations in mitochondrial function, including increased mitochondrial ROS generation and reduced ATP production [66]. Han et al. also revealed significantly higher levels of p53 and phospho-p53 in nuclei isolated from Sod1 ${ }^{-1-}$ livers [67]. Moreover, Wang et al. showed that Sod1 ablation led to increased p53 and phospho-p53 levels in islets [26]. In humans, decreased Sod1 expression and enhanced p53 expression were observed in AD-affected brain tissues [11, $68]$, osteoarthritic tissues $[69,70]$, bones in older individuals $[71,72]$, and tissues in infertility patients [73, 74]. Taken together, these data suggest that cytoplasmic SOD1 loss induced the DNA damage response, which was associated with p53 upregulation, resulting in age-related pathologies.

\section{Conclusion and Perspective}

In the present review, we introduced various organ and tissue phenotypes of Sod1 ${ }^{-1-}$ mice. Using Sod1 ${ }^{-/-}$mice, we and other groups have demonstrated that Sodl deficiency enhances the intracellular $\mathrm{O}_{2}{ }^{--}$production and oxidative damage, resulting in global, age-related pathological changes, including changes in the brain, eyes, ears, blood, bones, skin, muscles, pancreas, liver, and ovaries during aging. Antioxidant treatment prevented or improved the pathological changes in Sod1 $1^{-1-}$ organs and tissues. Interestingly, Sodl does not appear to interact with other major antioxidant enzymes, such as Sod2, Sod3, and Gpxl, in terms of the organ and tissues pathologies, as demonstrated using double-knockout mice. These lines of evidence strongly indicated that Sodl plays a central role in maintaining the cellular redox balance and organ function in vivo. We also suggest that p53 plays a fundamental role in Sod1 ${ }^{-1-}$-related pathologies. Further analyses will be needed to clarify the contribution of p53 to the molecular signaling and age-related pathological changes induced by Sodl deficiency, including those using double mutant mice with Sod1 ${ }^{-/-}$and $p 53^{-/-}$.

\section{Conflict of Interests}

The authors declare that there is no conflict of interests regarding the publication of this paper.

\section{Acknowledgments}

This study was supported in part by the Program for the Promotion of Basic Research Activities for Innovative Biosciences and Grants-in-Aid for Scientific Research from the Ministry of Education, Culture, Sports, Science and Technology of Japan. The authors would like to thank Dr. Kazuma Murakami, from Kyoto University, and Dr. Daichi Morikawa, Dr. Keiji Kobayashi, Dr. Masato Koike, and Toshihiko Toda, from Chiba University, for their valuable technical assistance.

\section{References}

[1] C. López-Otín, M. A. Blasco, L. Partridge, M. Serrano, and G. Kroemer, "The hallmarks of aging," Cell, vol. 153, no. 6, pp. 11941217, 2013.

[2] T. Finkel and N. J. Holbrook, "Oxidants, oxidative stress and the biology of ageing," Nature, vol. 408, no. 6809, pp. 239-247, 2000.

[3] A. Okado-Matsumoto and I. Fridovich, "Subcellular distribution of superoxide dismutases (SOD) in rat liver. Cu, Zn-SOD in mitochondria," The Journal of Biological Chemistry, vol. 276, no. 42, pp. 38388-38393, 2001.

[4] Y. Li, T.-T. Huang, E. J. Carlson et al., "Dilated cardiomyopathy and neonatal lethality in mutant mice lacking manganese superoxide dismutase," Nature Genetics, vol. 11, no. 4, pp. 376381, 1995.

[5] T. Shimizu, H. Nojiri, S. Kawakami, S. Uchiyama, and T. Shirasawa, "Model mice for tissue-specific deletion of the manganese superoxide dismutase gene," Geriatrics and Gerontology International, vol. 10, supplement 1, pp. S70-S79, 2010.

[6] T. Shimizu, H. Nojiri, and T. Shirasawa, "Tissue-specific deletion of manganese superoxide dismutase in mice," in Systems Biology of Free Radicals and Antioxidants, I. Laher, Ed., pp. 475487, Springer, Berlin, Germany, 2014.

[7] L. M. Carlsson, J. Jonsson, T. Edlund, and S. L. Marklund, "Mice lacking extracellular superoxide dismutase are more sensitive to hyperoxia," Proceedings of the National Academy of Sciences of the United States of America, vol. 92, no. 14, pp. 6264-6268, 1995.

[8] A. G. Reaume, J. L. Elliott, E. K. Hoffman et al., "Motor neurons in $\mathrm{Cu} / \mathrm{Zn}$ superoxide dismutase-deficient mice develop normally but exhibit enhanced cell death after axonal injury," Nature Genetics, vol. 13, no. 1, pp. 43-47, 1996.

[9] S. Elchuri, T. D. Oberley, W. Qi et al., "CuZnSOD deficiency leads to persistent and widespread oxidative damage and hepatocarcinogenesis later in life," Oncogene, vol. 24, no. 3, pp. 367-380, 2005. 
[10] T.-T. Huang, M. Yasunami, E. J. Carlson et al., "Superoxidemediated cytotoxicity in superoxide dismutase-deficient fetal fibroblasts," Archives of Biochemistry and Biophysics, vol. 344, no. 2, pp. 424-432, 1997.

[11] K. Murakami, N. Murata, Y. Noda et al., "SOD1 (copper/zinc superoxide dismutase) deficiency drives amyloid $\beta$ protein oligomerization and memory loss in mouse model of Alzheimer disease," The Journal of Biological Chemistry, vol. 286, no. 52, pp. 44557-44568, 2011.

[12] K. Murakami, N. Murata, Y. Noda, K. Irie, T. Shirasawa, and T. Shimizu, "Stimulation of the amyloidogenic pathway by cytoplasmic superoxide radicals in an Alzheimer's disease mouse model," Bioscience, Biotechnology and Biochemistry, vol. 76, no. 6, pp. 1098-1103, 2012.

[13] Y. Imamura, S. Noda, K. Hashizume et al., "Drusen, choroidal neovascularization, and retinal pigment epithelium dysfunction in SOD1-deficient mice: a model of age-related macular degeneration," Proceedings of the National Academy of Sciences of the United States of America, vol. 103, no. 30, pp. 11282-11287, 2006.

[14] K. Hashizume, M. Hirasawa, Y. Imamura et al., "Retinal dysfunction and progressive retinal cell death in SOD1-deficient mice," The American Journal of Pathology, vol. 172, no. 5, pp. 1325-1331, 2008.

[15] A. Behndig, K. Karlsson, A. G. Reaume, M.-L. Sentman, and S. L. Marklund, "In vitro photochemical cataract in mice lacking copper-zinc superoxide dismutase," Free Radical Biology and Medicine, vol. 31, no. 6, pp. 738-744, 2001.

[16] T. Kojima, T. H. Wakamatsu, M. Dogru et al., "Age-related dysfunction of the lacrimal gland and oxidative stress: evidence from the Cu,Zn-superoxide dismutase-1 (Sod1) knockout mice," The American Journal of Pathology, vol. 180, no. 5, pp. 1879-1896, 2012.

[17] O. M. Ibrahim, M. Dogru, Y. Matsumoto et al., "Oxidative stress induced age dependent meibomian gland dysfunction in $\mathrm{cu}, \mathrm{zn}$ superoxide dismutase-1 (Sod1) knockout mice," PLoS ONE, vol. 9, no. 7, Article ID e99328, 2014.

[18] S. L. McFadden, D. Ding, A. G. Reaume, D. G. Flood, and R. J. Salvi, "Age-related cochlear hair cell loss is enhanced in mice lacking copper/zinc superoxide dismutase," Neurobiology of Aging, vol. 20, no. 1, pp. 1-8, 1999.

[19] K. K. Ohlemiller, S. L. McFadden, D.-L. Ding et al., "Targeted deletion of the cytosolic $\mathrm{Cu} / \mathrm{Zn}$-superoxide dismutase gene (Sod1) increases susceptibility to noise-induced hearing loss," Audiology and Neuro-Otology, vol. 4, no. 5, pp. 237-246, 1999.

[20] Y. Iuchi, F. Okada, K. Onuma et al., "Elevated oxidative stress in erythrocytes due to a SOD1 deficiency causes anaemia and triggers autoantibody production," Biochemical Journal, vol. 402, no. 2, pp. 219-227, 2007.

[21] H. Nojiri, Y. Saita, D. Morikawa et al., "Cytoplasmic superoxide causes bone fragility owing to low-turnover osteoporosis and impaired collagen cross-linking," Journal of Bone and Mineral Research, vol. 26, no. 11, pp. 2682-2694, 2011.

[22] D. Morikawa, H. Nojiri, Y. Saita et al., "Cytoplasmic reactive oxygen species and SOD1 regulate bone mass during mechanical unloading," Journal of Bone and Mineral Research, vol. 28, no. 11, pp. 2368-2380, 2013.

[23] K. Murakami, J. Inagaki, M. Saito et al., "Skin atrophy in cytoplasmic SOD-deficient mice and its complete recovery using a vitamin C derivative," Biochemical and Biophysical Research Communications, vol. 382, no. 2, pp. 457-461, 2009.
[24] S. Shibuya, K. Kinoshita, and T. Shimizu, "Protective effects of vitamin C derivatives on skin atrophy caused by Sod1 deficiency," in Handbook of Diet, Nutrition and the Skin, V. R. Preedy, Ed., pp. 351-364, Wageningen Academic, Gelderland, The Netherlands, 2012.

[25] F. L. Muller, W. Song, Y. Liu et al., "Absence of CuZn superoxide dismutase leads to elevated oxidative stress and acceleration of age-dependent skeletal muscle atrophy," Free Radical Biology and Medicine, vol. 40, no. 11, pp. 1993-2004, 2006.

[26] X. Wang, M. Z. Vatamaniuk, C. A. Roneker et al., "Knockouts of SOD1 and GPX1 exert different impacts on murine islet function and pancreatic integrity," Antioxidants and Redox Signaling, vol. 14, no. 3, pp. 391-401, 2011.

[27] G. Muscogiuri, A. B. Salmon, C. Aguayo-Mazzucato et al., "Genetic disruption of SOD1 gene causes glucose intolerance and impairs $\beta$-cell function," Diabetes, vol. 62 , no. 12 , pp. 42014207, 2013.

[28] S. Uchiyama, T. Shimizu, and T. Shirasawa, "CuZn-SOD deficiency causes ApoB degradation and induces hepatic lipid accumulation by impaired lipoprotein secretion in mice," Journal of Biological Chemistry, vol. 281, no. 42, pp. 31713-31719, 2006.

[29] Y.-S. Ho, M. Gargano, J. Cao, R. T. Bronson, I. Heimler, and R. J. Hutz, "Reduced fertility in female mice lacking copper-zinc superoxide dismutase," Journal of Biological Chemistry, vol. 273, no. 13, pp. 7765-7769, 1998.

[30] M. M. Matzuk, L. Dionne, Q. Guo, T. R. Kumar, and R. M. Lebovitz, "Ovarian function in superoxide dismutase 1 and 2 knockout mice," Endocrinology, vol. 139, no. 9, pp. 4008-4011, 1998.

[31] Y. Noda, K. Ota, T. Shirasawa, and T. Shimizu, "Copper/Zinc superoxide dismutase insufficiency impairs progesterone secretion and fertility in female mice," Biology of Reproduction, vol. 86, no. 1, pp. 1-8, 2012.

[32] G. G. Kovacs, H. Adle-Biassette, I. Milenkovic, S. Cipriani, J.van Scheppingen, and E. Aronica, "Linking pathways in the developing and aging brain with neurodegeneration," Neuroscience, vol. 269, pp. 152-172, 2014.

[33] M. A. Ansari and S. W. Scheff, "Oxidative stress in the progression of alzheimer disease in the frontal cortex," Journal of Neuropathology and Experimental Neurology, vol. 69, no. 2, pp. 155-167, 2010.

[34] J. Ding and D. A. Sullivan, "Aging and dry eye disease," Experimental Gerontology, vol. 47, no. 7, pp. 483-490, 2012.

[35] B. J. Walters and J. Zuo, "Postnatal development, maturation and aging in the mouse cochlea and their effects on hair cell regeneration," Hearing Research, vol. 297, pp. 68-83, 2013.

[36] A. D. Walling and G. M. Dickson, "Hearing loss in older adults," American Family Physician, vol. 85, no. 12, pp. 1150-1156, 2012.

[37] D. E. Coling, K. C. Y. Yu, D. Somand et al., "Effect of SOD1 overexpression on age- and noise-related hearing loss," Free Radical Biology and Medicine, vol. 34, no. 7, pp. 873-880, 2003.

[38] R. L. Jilka, "The relevance of mouse models for investigating age-related bone loss in humans," Journals of Gerontology Series A: Biological Sciences and Medical Sciences, vol. 68, no. 10, pp. 1209-1217, 2013.

[39] X. Wang, E. A. Gillen, M. C. H. Van Der Meulen, and G. L. Xin, "Knockouts of Se-glutathione peroxidase-1 and $\mathrm{Cu}, \mathrm{Zn}$ superoxide dismutase exert different impacts on femoral mechanical performance of growing mice," Molecular Nutrition and Food Research, vol. 52, no. 11, pp. 1334-1339, 2008. 
[40] D. Morikawa, Y. Itoigawa, and H. Nojiri et al., "Contribution of oxidative stress to the degeneration of rotator cuff entheses," Journal of Shoulder and Elbow Surgery, vol. 23, no. 5, pp. 628635, 2014.

[41] J. Khavkin and D. A. F. Ellis, "Aging skin: histology, physiology, and pathology," Facial Plastic Surgery Clinics of North America, vol. 19, no. 2, pp. 229-234, 2011.

[42] E. Kohl, J. Steinbauer, M. Landthaler, and R.-M. Szeimies, "Skin ageing," Journal of the European Academy of Dermatology and Venereology, vol. 25, no. 8, pp. 873-884, 2011.

[43] K. Watanabe, S. Shibuya, H. Koyama et al., "Sod1 loss induces intrinsic superoxide accumulation leading to p53-mediated growth arrest and apoptosis," International Journal of Molecular Sciences, vol. 14, no. 6, pp. 10998-11010, 2013.

[44] R. A. Fielding, B. Vellas, W. J. Evans et al., "Sarcopenia: an undiagnosed condition in older adults. Current consensus definition: prevalence, etiology, and consequences. International working group on sarcopenia," Journal of the American Medical Directors Association, vol. 12, no. 4, pp. 249-256, 2011.

[45] Y. C. Jang, M. S. Lustgarten, Y. Liu et al., "Increased superoxide in vivo accelerates age-associated muscle atrophy through mitochondrial dysfunction and neuromuscular junction degeneration," FASEB Journal, vol. 24, no. 5, pp. 1376-1390, 2010.

[46] Y. Zhang, C. Davis, G. K. Sakellariou et al., "CuZnSOD gene deletion targeted to skeletal muscle leads to loss of contractile force but does not cause muscle atrophy in adult mice," FASEB Journal, vol. 27, no. 9, pp. 3536-3548, 2013.

[47] G. K. Sakellariou, C. S. Davis, Y. Shi et al., "Neuron-specific expression of CuZnSOD prevents the loss of muscle mass and function that occurs in homozygous CuZnSOD-knockout mice," The FASEB Journal, vol. 28, no. 4, pp. 1666-1681, 2014.

[48] D. Elahi, D. C. Muller, J. M. Egan, R. Andres, J. Veldhuis, and G. S. Meneilly, "Glucose tolerance, glucose utilization and insulin secretion in ageing," Novartis Foundation Symposium, vol. 242, pp. 222-246, 2002.

[49] A. Anantharaju, A. Feller, and A. Chedid, "Aging liver: a review," Gerontology, vol. 48, no. 6, pp. 343-353, 2002.

[50] L. Wang, Z. Jiang, and X. G. Lei, "Knockout of SOD1 alters murine hepatic glycolysis, gluconeogenesis, and lipogenesis," Free Radical Biology and Medicine, vol. 53, no. 9, pp. 1689-1696, 2012.

[51] Y. Kondo, Y. Inai, Y. Sato et al., "Senescence marker protein 30 functions as gluconolactonase in L-ascorbic acid biosynthesis, and its knockout mice are prone to scurvy," Proceedings of the National Academy of Sciences of the United States of America, vol. 103, no. 15, pp. 5723-5728, 2006.

[52] Y. Kondo, H. Masutomi, Y. Noda et al., "Senescence marker protein-30/superoxide dismutase 1 double knockout mice exhibit increased oxidative stress and hepatic steatosis," FEBS Open Bio, vol. 4, pp. 522-532, 2014.

[53] X. G. Lei, J.-H. Zhu, J. P. McClung, M. Aregullin, and C. A. Roneker, "Mice deficient in $\mathrm{Cu}, \mathrm{Zn}$-superoxide dismutase are resistant to acetaminophen toxicity," Biochemical Journal, vol. 399, no. 3, pp. 455-461, 2006.

[54] J.-H. Zhu, X. Zhang, C. A. Roneker et al., "Role of copper,zincsuperoxide dismutase in catalyzing nitrotyrosine formation in murine liver," Free Radical Biology and Medicine, vol. 45, no. 5, pp. 611-618, 2008.

[55] M. Szafarowska and M. Jerzak, "Ovarian aging and infertility," Ginekologia Polska, vol. 84, no. 4, pp. 298-304, 2013.
[56] N. Kimura, S. Tsunoda, Y. Iuchi, H. Abe, K. Totsukawa, and J. Fujii, "Intrinsic oxidative stress causes either 2-cell arrest or cell death depending on developmental stage of the embryos from SOD1-deficient mice," Molecular Human Reproduction, vol. 16, no. 7, pp. 441-451, 2010.

[57] R. M. Colven and S. R. Pinnell, "Topical vitamin C in aging," Clinics in Dermatology, vol. 14, no. 2, pp. 227-234, 1996.

[58] K. Murakami, N. Murata, Y. Ozawa et al., "Vitamin C restores behavioral deficits and amyloid- $\beta$ oligomerization without affecting plaque formation in a mouse model of alzheimer's disease," Journal of Alzheimer's Disease, vol. 26, no. 1, pp. 7-18, 2011.

[59] T. Ikegami, Y.-I. Suzuki, T. Shimizu, K.-I. Isono, H. Koseki, and T. Shirasawa, "Model mice for tissue-specific deletion of the manganese superoxide dismutase (MnSOD) gene," Biochemical and Biophysical Research Communications, vol. 296, no. 3, pp. 729-736, 2002.

[60] M.-L. Sentman, M. Granström, H. Jakobson, A. Reaume, S. Basu, and S. L. Marklund, "Phenotypes of mice lacking extracellular superoxide dismutase and copper- and zinc-containing superoxide dismutase," Journal of Biological Chemistry, vol. 281, no. 11, pp. 6904-6909, 2006.

[61] H. Fujita, H. Fujishima, K. Takahashi et al., "SOD1, but not SOD3, deficiency accelerates diabetic renal injury in C57BL/6Ins2Akita diabetic mice," Metabolism: Clinical and Experimental, vol. 61, no. 12, pp. 1714-1724, 2012.

[62] J.-H. Zhu and X. G. Lei, "Lipopolysaccharide-induced hepatic oxidative injury is not potentiated by knockout of GPX1 and SOD1 in mice," Biochemical and Biophysical Research Communications, vol. 404, no. 1, pp. 559-563, 2011.

[63] S. K. Wang, J. D. Weaver, S. Zhang, and X. G. Lei, "Knockout of SOD1 promotes conversion of selenocysteine to dehydroalanine in murine hepatic GPX1 protein," Free Radical Biology and Medicine, vol. 51, no. 1, pp. 197-204, 2011.

[64] S. Ditch and T. T. Paull, "The ATM protein kinase and cellular redox signaling: beyond the DNA damage response," Trends in Biochemical Sciences, vol. 37, no. 1, pp. 15-22, 2012.

[65] L. Erker, R. Schubert, S. Elchuri et al., "Effect of the reduction of superoxide dismutase 1 and 2 or treatment with $\alpha$-tocopherol on tumorigenesis in Atm-deficient mice," Free Radical Biology and Medicine, vol. 41, no. 4, pp. 590-600, 2006.

[66] F. L. Muller, W. Song, Y. C. Jang et al., "Denervation-induced skeletal muscle atrophy is associated with increased mitochondrial ROS production," American Journal of Physiology. Regulatory Integrative and Comparative Physiology, vol. 293, no. 3, pp. R1159-R1168, 2007.

[67] E. S. Han, F. L. Muller, V. I. Pérez et al., "The in vivo gene expression signature of oxidative stress," Physiol Genomics, vol. 34, no. 1, pp. 112-126, 2008.

[68] G. Cenini, R. Sultana, M. Memo, and D. A. Butterfield, "Elevated levels of pro-apoptotic p53 and its oxidative modification by the lipid peroxidation product, HNE, in brain from subjects with amnestic mild cognitive impairment and Alzheimer's disease," Journal of Cellular and Molecular Medicine, vol. 12, no. 3, pp. 987-994, 2008.

[69] S. Zhou, J. S. Greenberger, M. W. Epperly et al., "Age-related intrinsic changes in human bone-marrow-derived mesenchymal stem cells and their differentiation to osteoblasts," Aging Cell, vol. 7, no. 3, pp. 335-343, 2008.

[70] J. L. Scott, C. Gabrielides, R. K. Davidson et al., "Superoxide dismutase downregulation in osteoarthritis progression and 
end-stage disease," Annals of the Rheumatic Diseases, vol. 69, no. 8, pp. 1502-1510, 2010.

[71] M. Almeida, "Aging mechanisms in bone," BoneKEy Reports, vol. 1, article 102, 2012.

[72] D. Maggio, M. Barabani, M. Pierandrei et al., "Marked decrease in plasma antioxidants in aged osteoporotic women: results of a cross-sectional study," Journal of Clinical Endocrinology and Metabolism, vol. 88, no. 4, pp. 1523-1527, 2003.

[73] L. R. Fraga, C. G. Dutra, J. A. Boquett et al., "p53 signaling pathway polymorphisms associated to recurrent pregnancy loss," Molecular Biology Reports, vol. 41, no. 3, pp. 1871-1877, 2014.

[74] C. Tatone, M. C. Carbone, S. Falone et al., "Age-dependent changes in the expression of superoxide dismutases and catalase are associated with ultrastructural modifications in human granulosa cells," Molecular Human Reproduction, vol. 12, no. 11, pp. 655-660, 2006. 

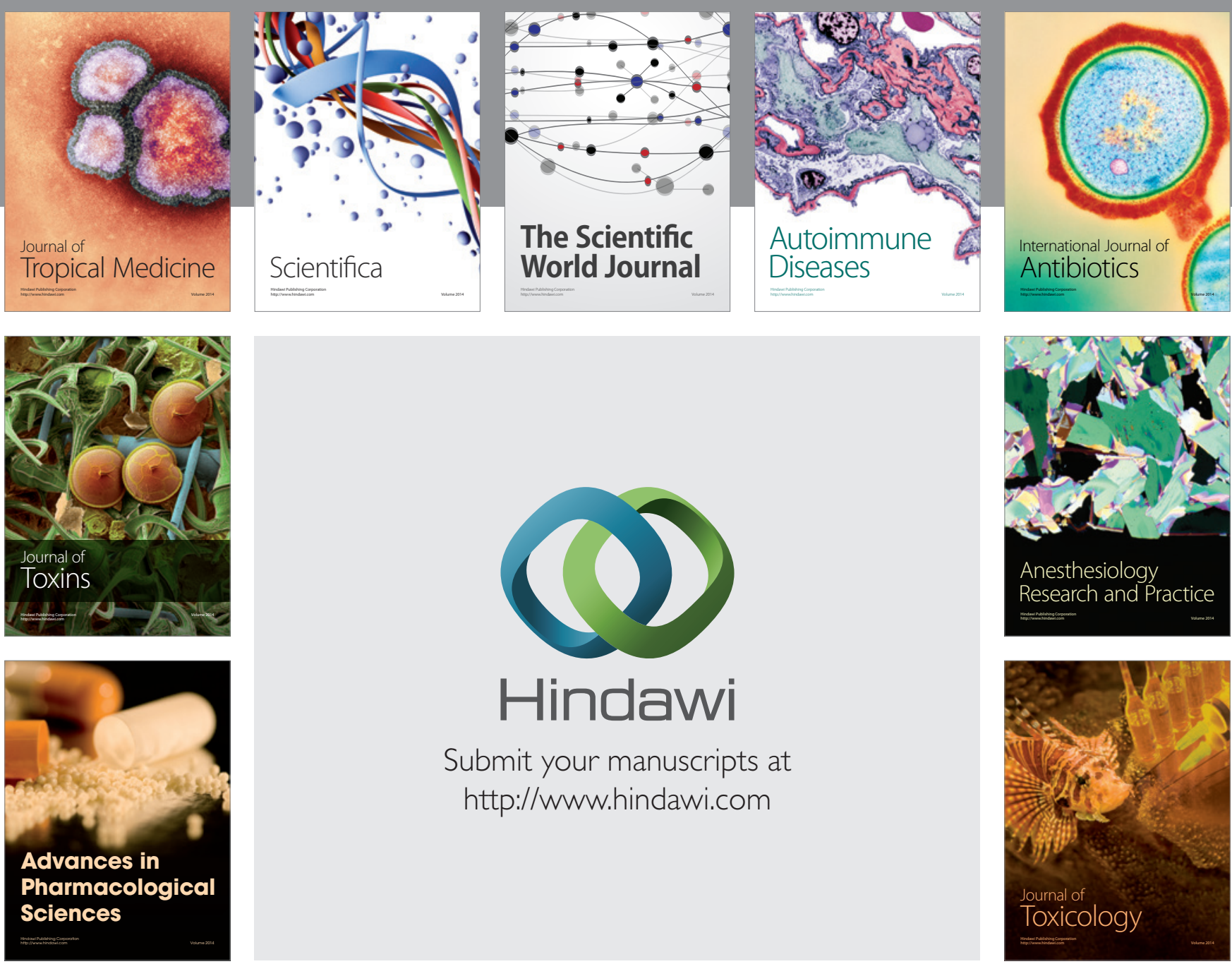

\section{Hindawi}

Submit your manuscripts at

http://www.hindawi.com
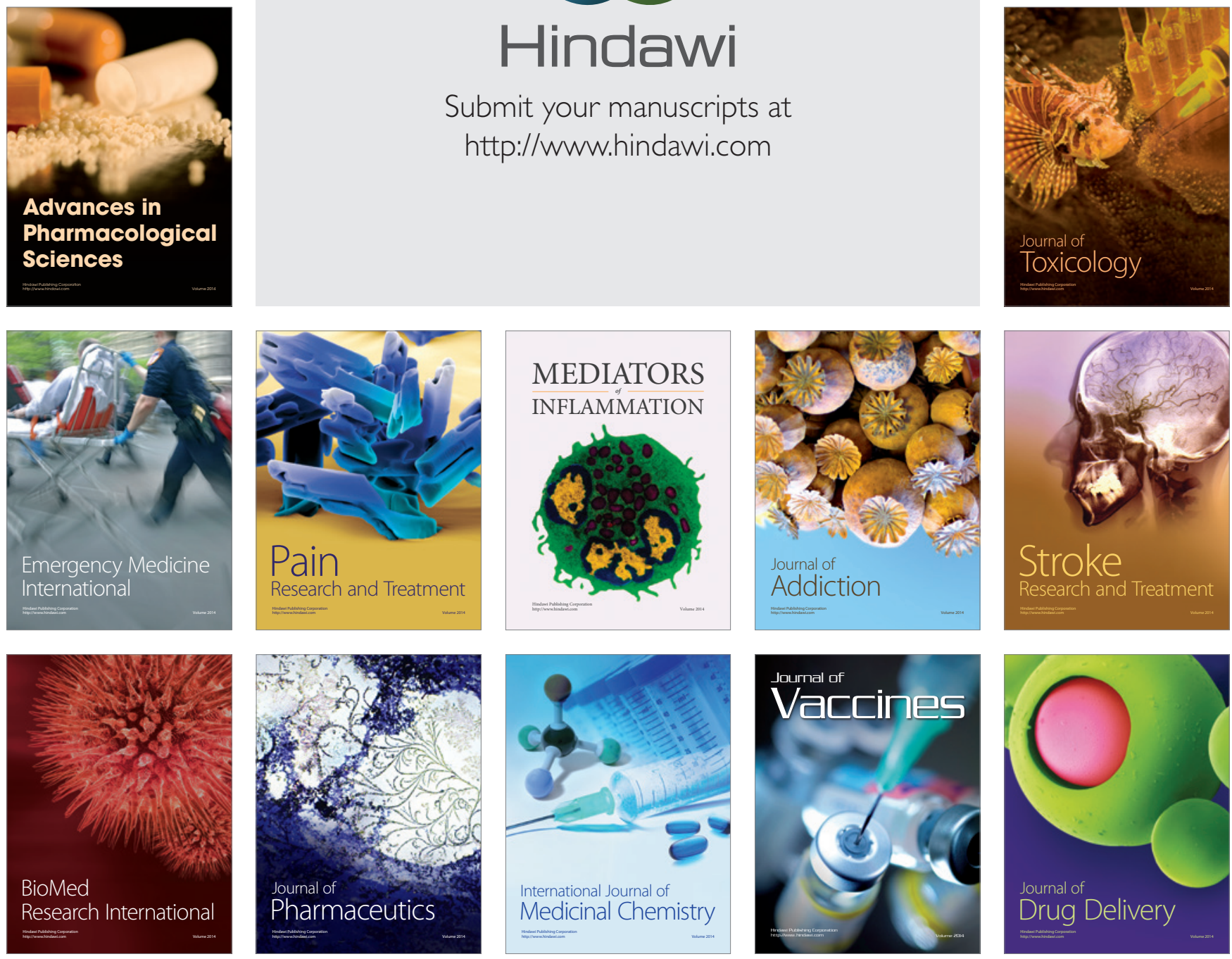